\title{
Supporting Information for \\ All-Dielectric Silicon Nanogap Antennas \\ to Enhance the Fluorescence of Single Molecules
}

Raju Regmi, ${ }^{1,2}$ Johann Berthelot, ${ }^{1}$ Pamina M. Winkler, ${ }^{2}$ Mathieu Mivelle, ${ }^{3}$ Julien Proust, ${ }^{1}$

Frédéric Bedu, ${ }^{4}$ Igor Ozerov, ${ }^{4}$ Thomas Begou, ${ }^{1}$ Julien Lumeau, ${ }^{1}$ Hervé Rigneault, ${ }^{1}$ Maria F. Garcia-Parajó, ${ }^{2,5}$ Sébastien Bidault, ${ }^{6}$ Jérôme Wenger, $^{1 *}$ and Nicolas Bonod ${ }^{1 *}$

${ }^{1}$ Aix Marseille Univ, CNRS, Centrale Marseille, Institut Fresnel, Marseille, France

${ }^{2}$ ICFO-Institut de Ciencies Fotoniques, The Barcelona Institute of Science and Technology, 08860 Barcelona, Spain

${ }^{3}$ Université Pierre et Marie Curie, CNRS, Institut des NanoSciences de Paris, UMR 7588, 75005 Paris, France

${ }^{4}$ Aix Marseille Univ, CNRS, CINAM, Marseille, France

${ }^{5}$ ICREA, Pg. Lluís Companys 23, 08010 Barcelona, Spain

${ }^{6}$ ESPCI Paris, PSL Research University, CNRS, INSERM, Institut Langevin, 75005 Paris, France

*Email: jerome.wenger@fresnel.fr, nicolas.bonod@fresnel.fr

This document contains the following supporting information:

1. Silicon nanoantenna fabrication

2. Numerical simulation of electric field intensity enhancement in silicon dimer nanoantennas

3. Comparison with electric field intensity enhancement in gold dimer nanoantennas

4. Overlap between the antenna resonance and the fluorescence absorption and emission spectra

5. Luminescence background when no fluorescent dye is present

6. Quantum yield enhancement for $20 \mathrm{~nm}$ silicon nanogap antenna

7. FCS analysis of crystal violet fluorescence traces on silicon antenna

8. Comparison of decay rates for dipolar source parallel and perpendicular to the gap

9. Radiation patterns for a dipolar source parallel and perpendicular to the gap

10. Reference fluorescence decay kinetics on confocal setup 


\section{Silicon nanoantenna fabrication}

The all-silicon nanogap antennas are fabricated by creating a mask with electron beam lithography in a poly(methyl methacrylate) PMMA layer coated on a $60 \mathrm{~nm}$ thick silicon layer. The amorphous silicon layer is deposited on a $150 \mu \mathrm{m}$ thick silica coverslip by Plasma Assisted Reactive Magnetron Sputtering (PARMS) using a Buhler HELIOS machine. The layers are obtained by the sputtering of two silicon targets with a plasma of Argon (30 sccm) excited with a mid frequency source ( $4500 \mathrm{~W}$ at 40 $\mathrm{kHz})$ at low pressure $\left(5.10^{-4} \mathrm{mbar}\right)$. The precise control of the thickness of the deposited layer is carried out using an in-situ optical monitoring system. This system measures the evolution of the monochromatic transmission during deposition. The monitoring wavelength is chosen to be equal at $1500 \mathrm{~nm}$. At this wavelength the absorption of silicon layers is negligible and the real part of the refractive index is equal to 3.695. Deposition is stopped by using a trigger point technique, i.e. when the transmission of the deposited layer on a fused silica test glass is equal to $46.3 \%$. Precision and uniformity of the thickness is better than $0.5 \%$. After deposition, the samples are cleaned in successive ultrasound baths in acetone and isopropyl alcohol (IPA, propan-2-ol), dried under clean nitrogen flow and exposed to oxygen plasma at $150^{\circ} \mathrm{C}$ (Nanoplas, France) for 10 minutes. A 60-70 nm thick commercial PMMA positive e-beam resist (ARP-679, Allresist, Germany) diluted at $2 \%$ in ethyl lactate solvent is spin-coated at $4000 \mathrm{rpm}$ onto the silicon surface and baked on a hotplate to remove the remaining solvent and harden the PMMA layer. A second conducting polymer layer (SX AR-PC 5000/90.1 from Allresist, Germany) of thickness of $30 \mathrm{~nm}$ is spin-coated on the first PMMA ebeam resist to reduce the sample charging and to increase the EBL resolution. The samples are exposed to electron beam using an EBL tool (Pioneer, Raith, Germany) equipped with field emission gun (FEG) electron source (acceleration voltage of $20 \mathrm{kV}$, apertures of 7.5 and $10 \mu \mathrm{m}$, beam current of 18 to $30 \mathrm{pA}$ ). We varied both the designed distances between the features and the expose dose in order to finely tune the gap between the particles. The nominal dose is $120 \mu \mathrm{c} / \mathrm{cm}^{2}$. Several dose coefficients are tested $(0.8,0.9)$. For example, the $20 \mathrm{~nm}$ nanogap antennas are obtained with a dose coefficient of 0.9 corresponding to a dose of $0.9 \times 120 \mu \mathrm{C} / \mathrm{cm}^{2}$. After exposure, the conducting layer is removed in deionised water, and the PMMA is developed in a commercial solution (AR 600-55 from Allresist) during 60 seconds. A $15 \mathrm{~nm}$ thick metal nickel mask is then evaporated on the sample under vacuum (Auto 306 tool from Edwards, UK). After metallization, a lift-off process is performed in ethyl lactate using ultrasonic cleaning bath. During the lift-off, the remaining e-beam resist and the excess of nickel are removed. Finally, the sample is rinsed in deionized water and dried under nitrogen flow. The unprotected areas are etched in a RIE tool (MG-200, Plassys, France) by a gas mixture containing $\mathrm{SF}_{6}, \mathrm{O}_{2}$ and $\mathrm{CHF}_{3}$ (respective fluxes 20,8 and $5 \mathrm{sccm}$ ) for 10 seconds, alternated with a pure $\mathrm{O}_{2}$ plasma for 5 seconds. Excited $\mathrm{SF}_{6}$ is known to efficiently etch silicon and the admixture of $\mathrm{CHF}_{3}$ gas is used to passivate the vertical feature walls and to etch the silicon oxide on the very reactive silicon surfaces during the process. This process offers a very good etching anisotropy and nearly vertical walls. After RIE, the remaining nickel is removed chemically in the acid solution of $\mathrm{HCl}$ and $\mathrm{FeCl}_{3}$. Finally, the samples are rinsed in deionized water and dried under nitrogen flow. Scanning 
electron microscopy images are performed on a FEI DB235 microscope with field emission gun and 5 $\mathrm{kV}$ acceleration voltage, providing about 4-5 $\mathrm{nm}$ spatial resolution.

a
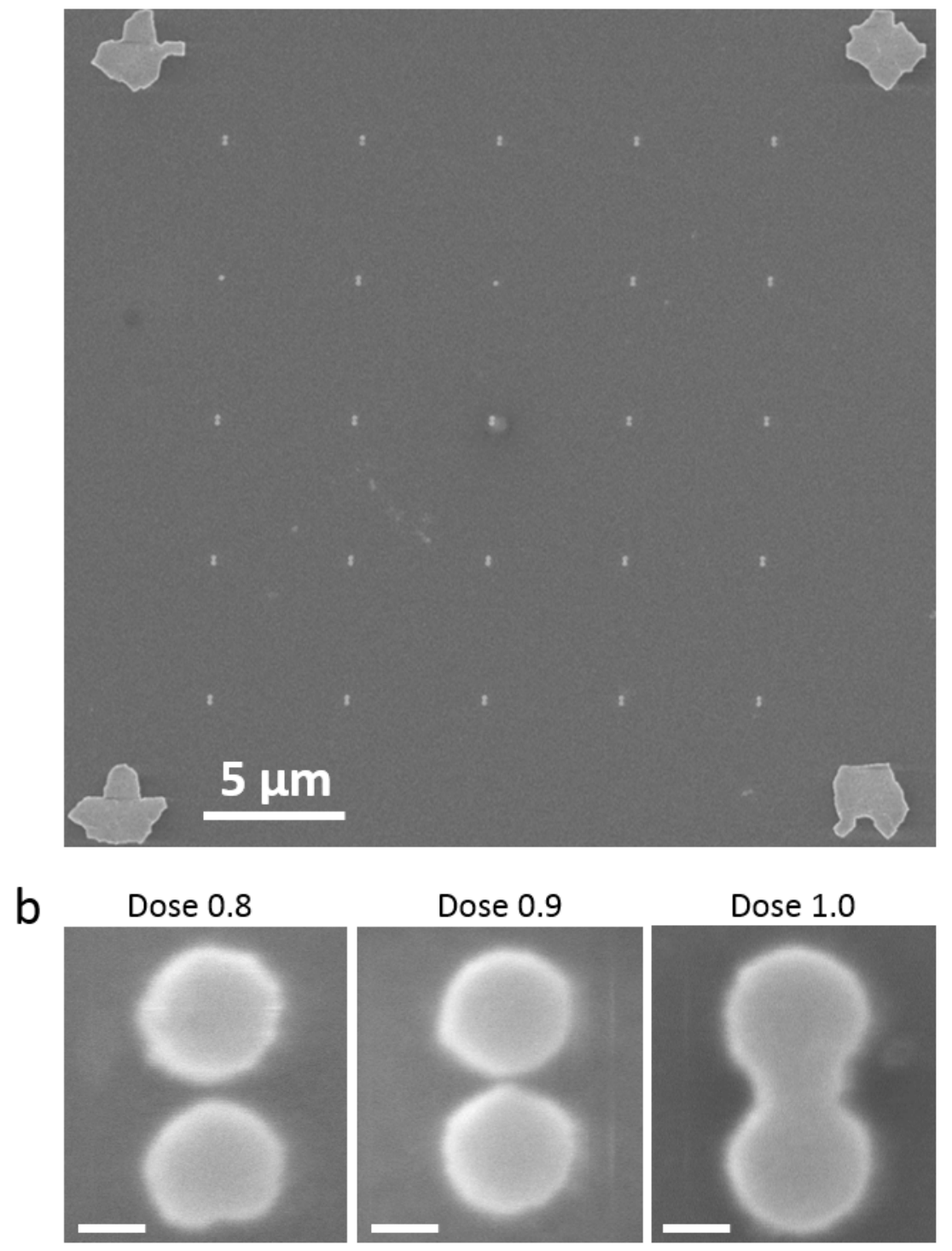

Figure S1. (a) SEM image of an array of silicon dimer antennas with gaps measured around $20 \mathrm{~nm}$. b) SEM images of silicon dimers in function of the e-beam exposure dose coefficient allowing to fabricate the $30 \mathrm{~nm}$ gap (dose 0.8 ) and the $20 \mathrm{~nm}$ gap antennas (dose 0.9). For higher doses, the antennas are bridged. The scale bar is $100 \mathrm{~nm}$. 


\section{Numerical simulation of electric field intensity enhancement in silicon dimer nanoantennas}
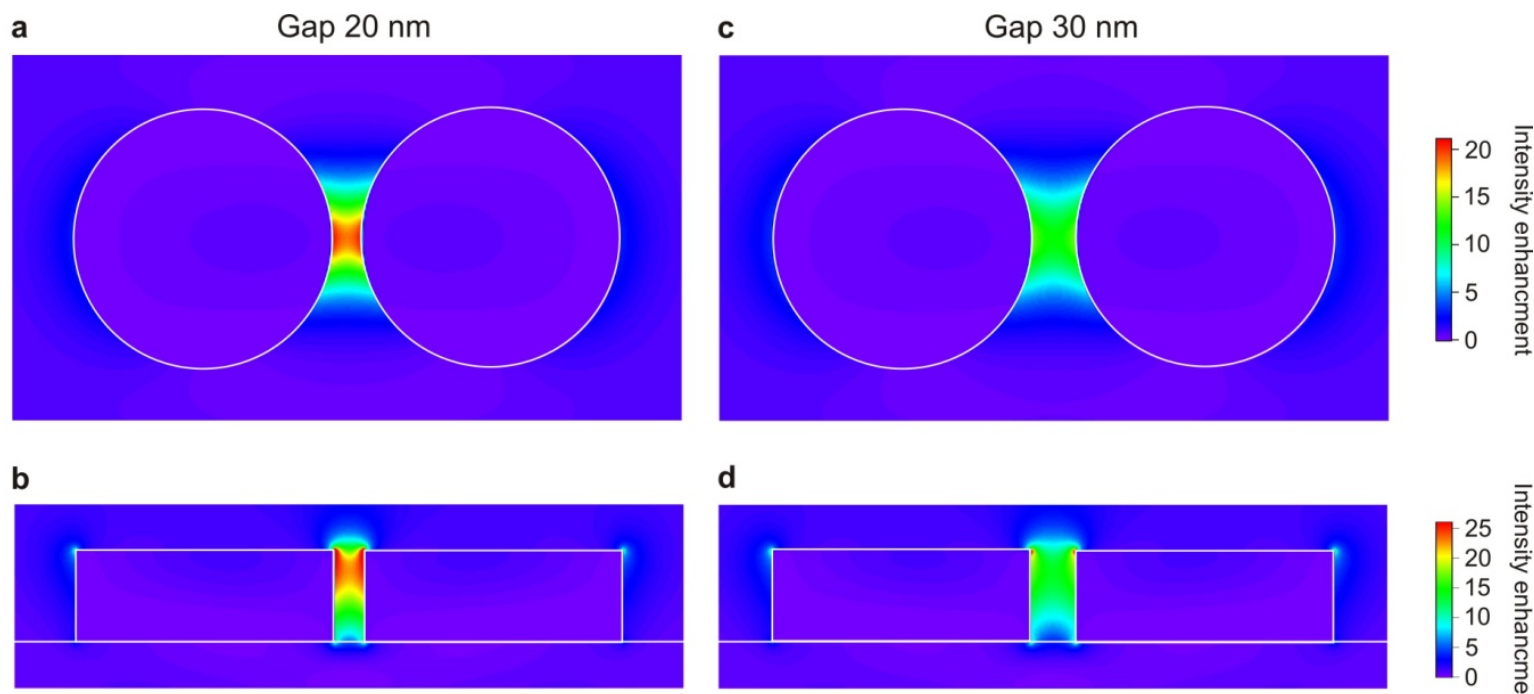

d

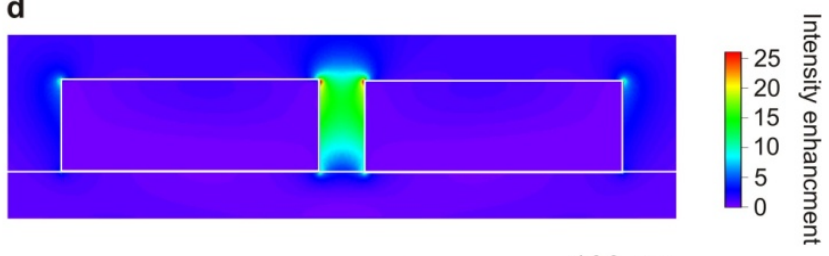

Figure S2. FDTD simulations of the electric field intensity distributions around the silicon dimer of $170 \mathrm{~nm}$ diameter with $20 \mathrm{~nm}$ gap $(\mathrm{a}, \mathrm{b})$ and $30 \mathrm{~nm}$ gap $(\mathrm{c}, \mathrm{d})$. The silicon antenna is illuminated at $\lambda=$ $633 \mathrm{~nm}$ in normal incidence from the glass substrate with a linear electric field polarized parallel to the dimer axis. The images in $(a, c)$ correspond to the horizontal plane crossing the center height of the dimer, while the images $(b, d)$ are vertical cross-sections along the main dimer axis. The color scales are common for $(a, c)$ and $(b, d)$ to ease comparison between the gap sizes. 


\section{Comparison with electric field intensity enhancement in gold dimer nanoantennas}
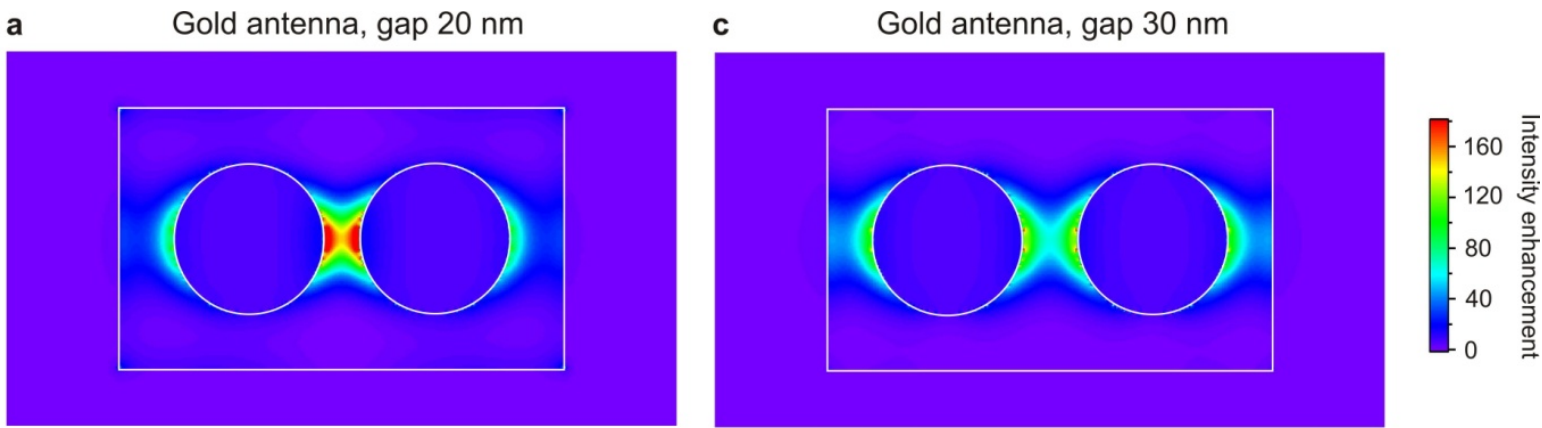

b

d
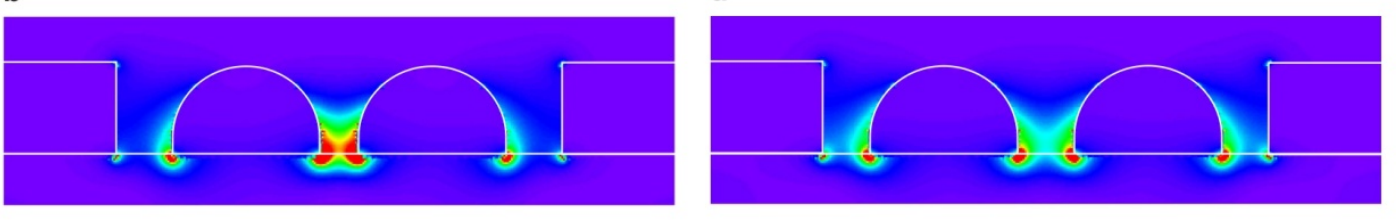

$80 \mathrm{~nm}$

$80 \mathrm{~nm}$

Figure S3. FDTD simulations of the electric field intensity distributions around the gold antenna of 80 $\mathrm{nm}$ diameter particles with $20 \mathrm{~nm}$ gap $(\mathrm{a}, \mathrm{b})$ and $30 \mathrm{~nm}$ gap $(\mathrm{c}, \mathrm{d})$. The gold antenna is illuminated at $\lambda$ $=633 \mathrm{~nm}$ in normal incidence from the glass substrate with a linear electric field polarized parallel to the dimer axis. The images in $(a, c)$ correspond to the horizontal plane located inside the antenna at 7 $\mathrm{nm}$ from the gold-glass interface, while the images $(b, d)$ are vertical cross-sections along the main dimer axis. The color scales are common for $(a, c)$ and $(b, d)$ to ease comparison between the gap sizes. 
a

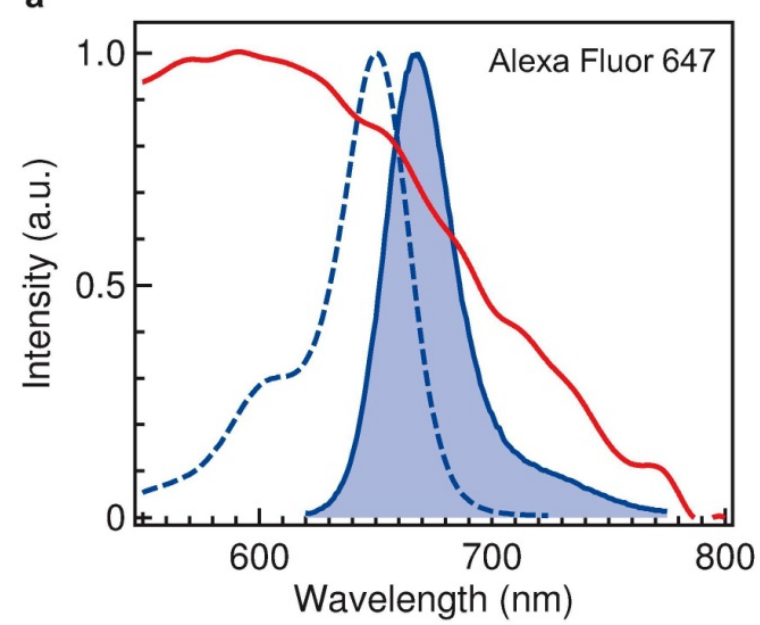

b

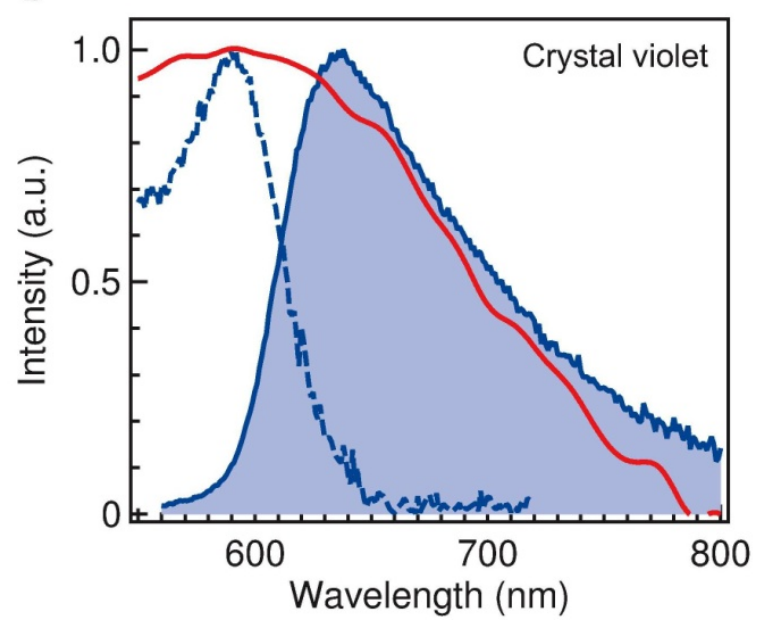

Figure S4. Spectral overlap between the dark-field scattering spectrum for the silicon antenna with $20 \mathrm{~nm}$ gap (red line) and the excitation (dashed lines) and emission (solid shadowed lines) fluorescence spectra for Alexa Fluor 647 (a) and Crystal Violet (b). 


\section{Luminescence background when no fluorescent dye is present}
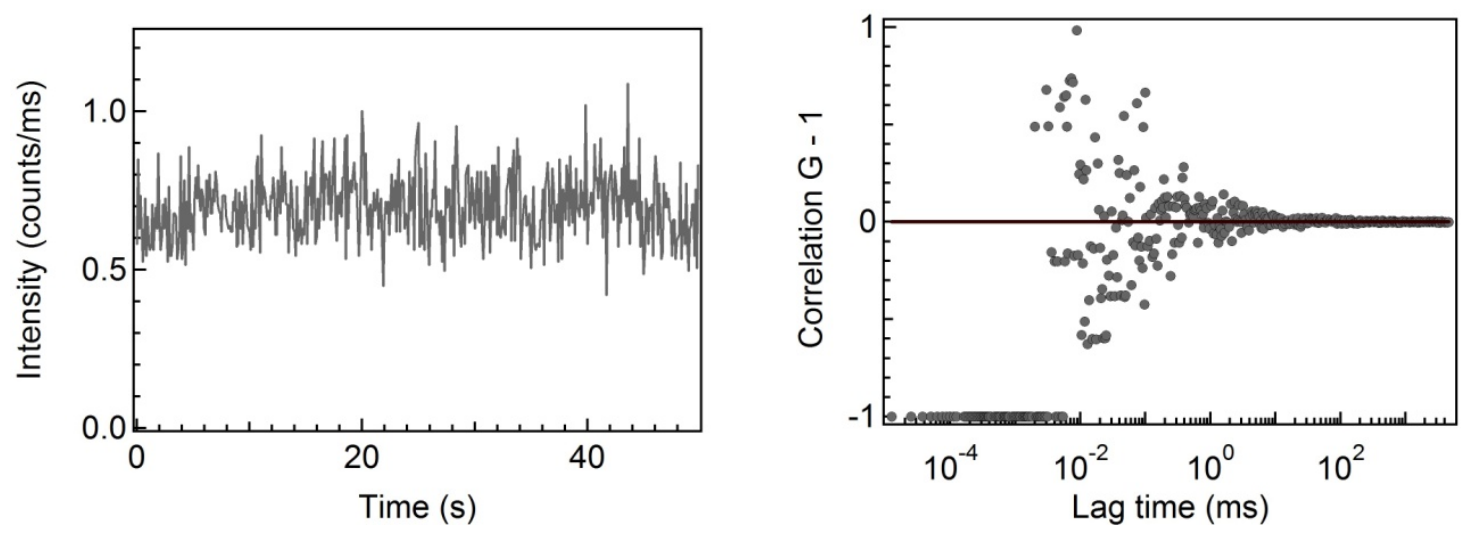

Figure S5. Intensity trace and correlation function on a silicon antenna with $20 \mathrm{~nm}$ gap size. No fluorescent molecule is used in this experiment to record the level of luminescence background. The $10 \mu \mathrm{W}$ excitation power at $633 \mathrm{~nm}$ is similar to the conditions used in Fig. 3a-c. No correlation is seen for lag times $>10 \mu \mathrm{s}$ as the curve is symmetrical around zero. For lag times $<10 \mu \mathrm{s}$, the extremely low detection rate does not enable to construct any correlation function, so the correlator output remains at the -1 level. 


\section{Quantum yield enhancement for $20 \mathrm{~nm}$ silicon nanogap antenna}
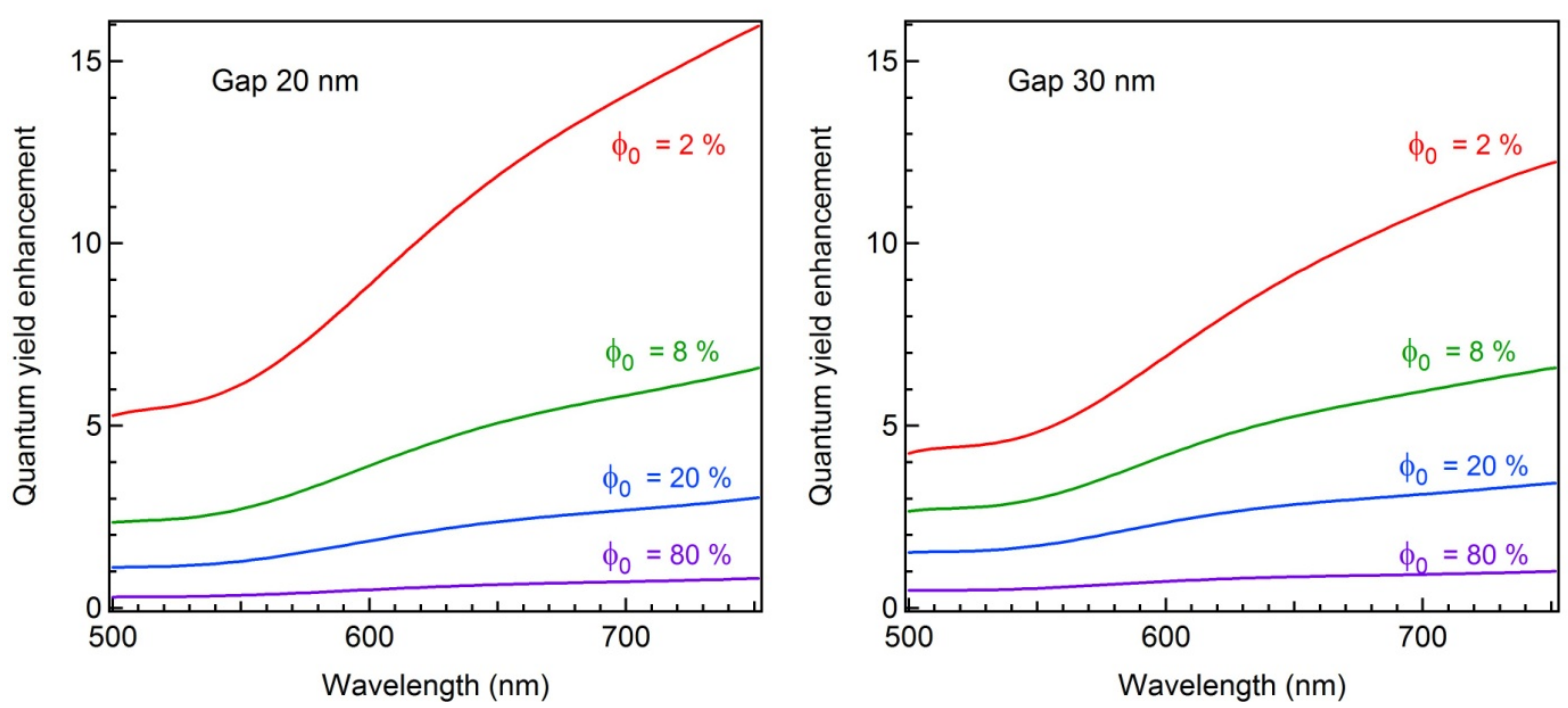

Figure S6. Quantum yield enhancement computed for a dipolar source oriented parallel $(X)$ to the silicon antenna main axis. The different values $\phi_{0}$ indicate the initial (intrinsic) quantum yield of the source. While no quantum yield enhancement is seen with a high efficiency emitter ( $\left.\phi_{0}>80 \%\right)$, using emitters with low intrinsic quantum yields maximizes the quantum yield enhancement. 


\section{FCS analysis of crystal violet fluorescence traces on silicon antenna}

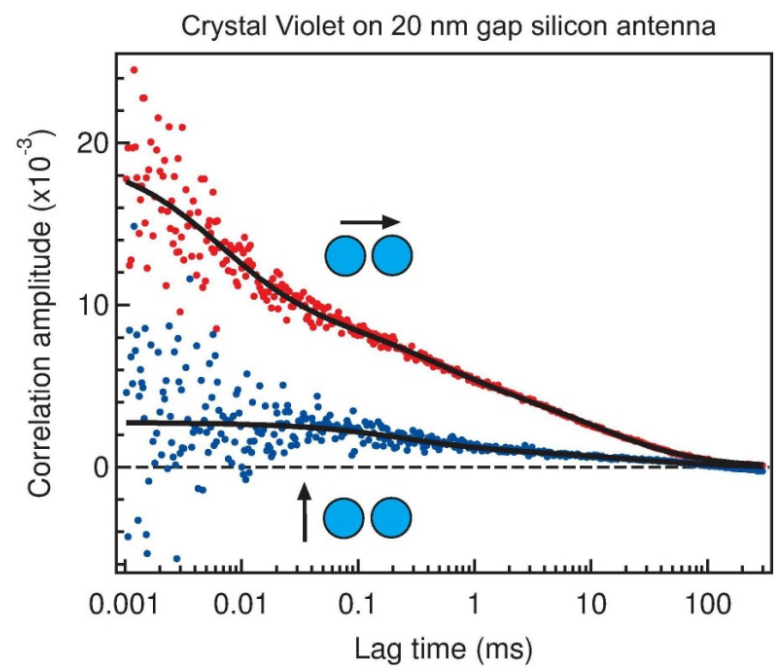

Figure S7. FCS correlation function for the fluorescence trace recorded with crystal violet on $20 \mathrm{~nm}$ silicon nanogap antenna with the excitation polarization parallel and perpendicular to the antenna main axis (the raw intensity traces are shown in Fig. $2 a \& 2 c$ ). The long correlation times in the millisecond range show that the fluorescence fluctuations for crystal violet are not limited by translational diffusion and indicate adsorption on the silicon surface. 


\section{Comparison of decay rates for a dipolar source parallel and perpendicular to the gap}
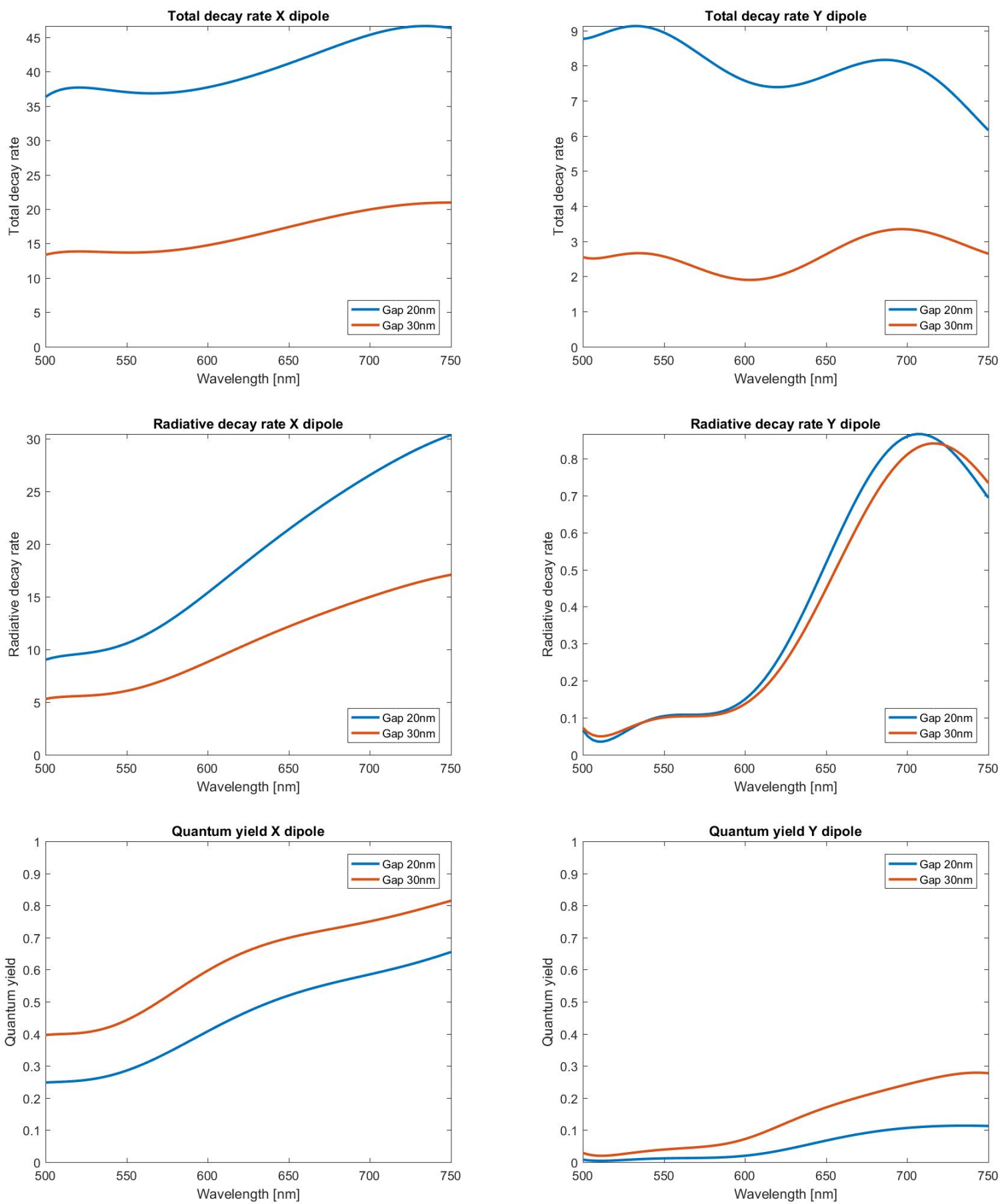

Figure S8. Comparison of total decay rate $\Gamma_{\text {tot }}$, radiative rate $\Gamma_{\text {rad }}$ and quantum yield $\Gamma_{\text {rad }} / \Gamma_{\text {tot }}$ for a dipole emitter located in the center of the silicon dimer antenna with orientation parallel (X, left column) or perpendicular ( $\mathrm{Y}$, right column) to the dimer main axis. All rates are normalized to the dipole's radiative rate in free space $\Gamma_{\mathrm{rad}, 0}$. The dipole with perpendicular orientation (Y) shows almost negligible radiative emission. 


\section{Radiation patterns for a dipolar source parallel and perpendicular to the gap}
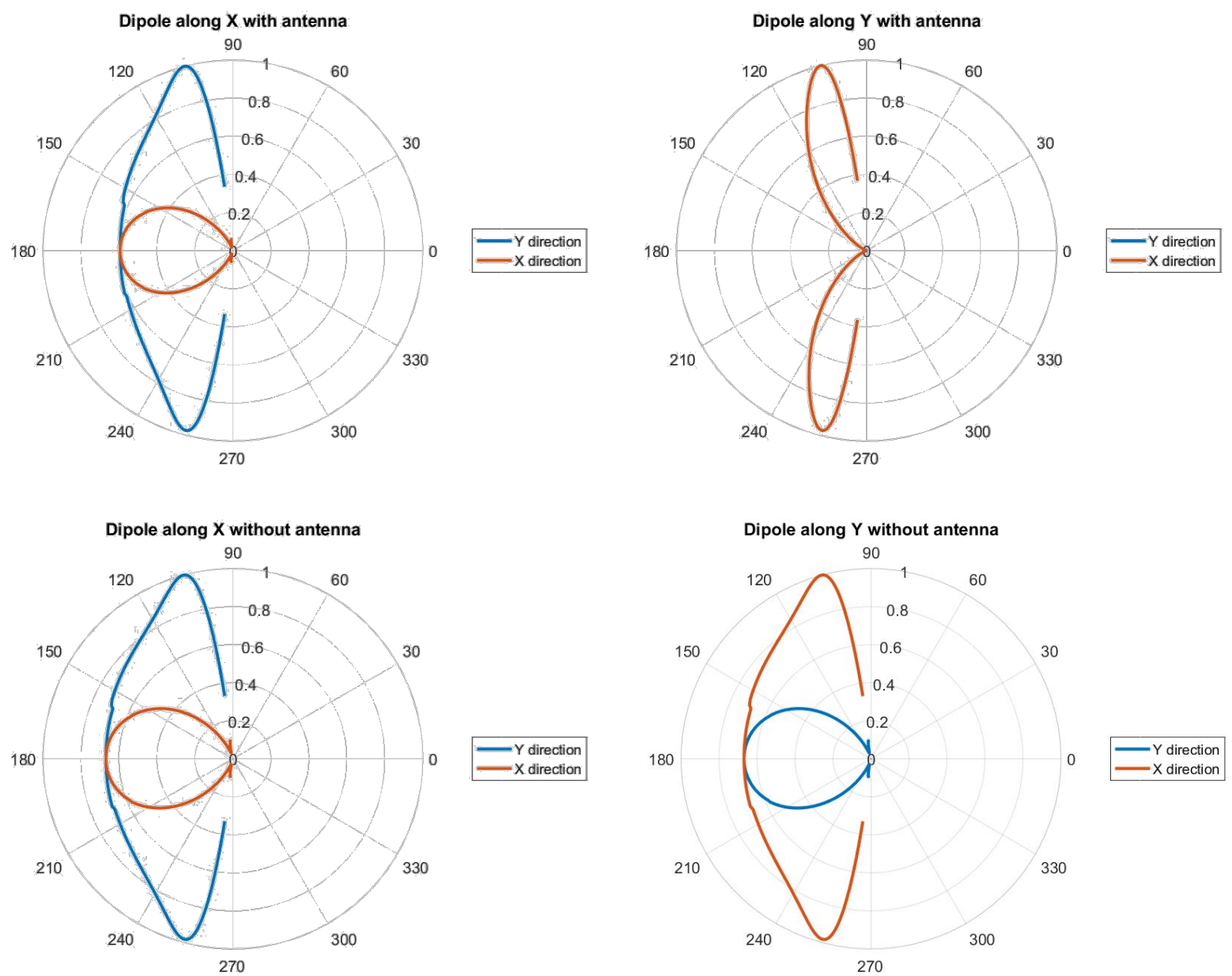

Figure S9. Comparison of radiation patterns for a dipole emitter located in the center of the silicon dimer antenna with orientation parallel ( $\mathrm{X}$, left column) or perpendicular ( $\mathrm{Y}$, right column) to the dimer main axis. The gap size is $20 \mathrm{~nm}$ and the emission wavelength is $670 \mathrm{~nm}$. The lower graphs show the reference radiation patterns for a dipole emitter in free space. While the antenna has negligible effect on the radiation pattern for the emitter with parallel $(\mathrm{X})$ orientation, the collection efficiency is reduced by $0.5 \mathrm{x}$ for the dipole with perpendicular $(\mathrm{Y})$ orientation. 


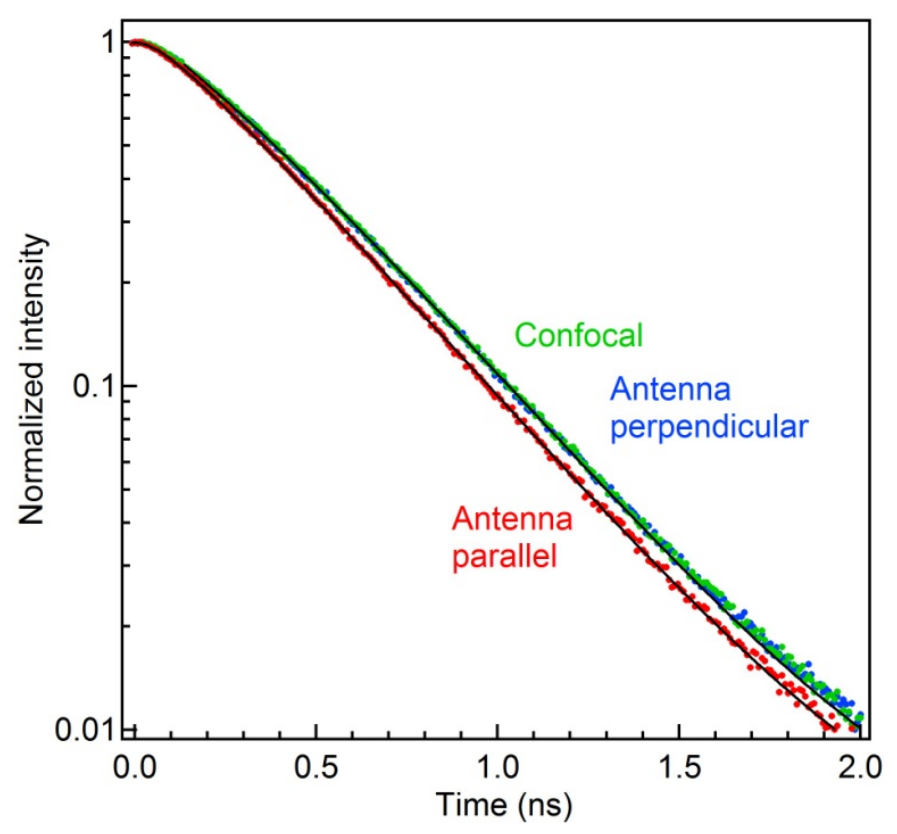

Figure S10. Normalized fluorescence decay traces of Alexa Fluor 647 with $200 \mathrm{mM}$ methyl viologen obtained on the confocal reference setup (green dots) and on a $20 \mathrm{~nm}$ gap silicon antenna with excitation light parallel (red) and perpendicular (blue) to the dimer axis. Black lines are numerical fits convoluted by the instrument response function. A single exponential decay with 350 ps lifetime is used to model the decay kinetics for the confocal reference and the antenna with perpendicular orientation. 